\title{
A Qualitative Analysis of Patients' Experiences during their Pathway to Psychiatric Care
}

\author{
Swetha Joju ${ }^{1}$, Manjula Simiyon ${ }^{2}$, Pradeep Thilakan ${ }^{3}$, Manikandan Mani $^{4}$
}

\begin{abstract}
Introduction: The pathway to care in psychiatry consists of a detailed and systematic description of sources of care used by the patients before seeking help from mental health professionals (MHPs).

Aim: To qualitatively evaluate the experiences of patients during various pathways taken by them through thematic analysis.

Materials and methods: This study was conducted at the Department of Psychiatry of Pondicherry Institute of Medical Sciences (PIMS), Puducherry. Those who had more than two encounters in the quantitative study were invited to participate. Interviews were conducted ensuring privacy, comfort, and confidentiality. Themes were analyzed using inductive coding method from the in-depth interviews.

Results: Themes related to participant's understanding about the illness, first symptom that triggered initiation, nature of encounters, reasons attributed by patients and healers, and remedies done were found. Some of the remedies prescribed by the traditional healers (THs) were violent and horrific.

Conclusion: This study throws light into the understanding of the pathways taken by psychiatric patients in South India and the need to promote awareness among general public including the magicoreligious healers.

Keywords: Pathway, Psychiatric care, Qualitative, Traditional healers.

Indian Journal of Private Psychiatry (2019): 10.5005/jp-journals-10067-0046
\end{abstract}

\section{INTRODUCTION}

Mental illnesses are commonly linked with a high burden of disease. The National Mental Health Survey ${ }^{1}$ conducted in India found that the overall prevalence of mental morbidity was $10.6 \%$ with a lifetime prevalence of $13.7 \%$. The pathways to care are the routes whereby people with mental disorders gain access to providers of mental health services. ${ }^{2}$ This consists of a detailed and systematic description of sources of care used by the patients before seeking help from metal health professionals (MHPs) and also of the factors that modify it. ${ }^{3}$ Only a limited proportion of patients with psychiatric disorders attend the mental healthcare facilities, and it is only when the condition becomes severe. ${ }^{4}$ Many patients are treated inadequately, which delays their presentation of illness and they become chronically ill, which depletes their resources. ${ }^{5}$

This is of special interest in India because of the shortage of access to trained MHPs and unique cultural diversity. Pathways to care in mental illnesses are more complicated and prolonged than other illnesses. Analyzing the pathway of care helps us to understand the health service utilization, recognizing reasons for the delay in reaching appropriate systems, and to strategically plan solutions. ${ }^{5}$

Culture exceptionally influences the mental health of people living in a society. India is a country with an amalgamation of vivid varieties of cultures and religious beliefs. Mental health problems, from the onset of illness to course and outcome, at every phase can be influenced by cultural beliefs. In India, blind beliefs and faith in the supernatural etiology of mental illness are highly prevalent among the majority of the population, especially in rural and tribal areas. ${ }^{6}$ Traditional healing has a long and significant history in India. ${ }^{7}$ In the Indian health system, mainly in rural India and partly in urban India, the first line of contact for any illness including mental illness is comprised of local healthcare providers, a majority of them are traditional healers $(\mathrm{THs}){ }^{8}$

\footnotetext{
${ }^{1-3}$ Department of Psychiatry, Pondicherry Institute of Medical Sciences, Puducherry, India

${ }^{4}$ Department of Community Medicine, Pondicherry Institute of
} Medical Sciences, Puducherry, India

Corresponding Author: Manjula Simiyon, Department of Psychiatry, Pondicherry Institute of Medical Sciences, Puducherry, India, Phone: +91 9677468423, e-mail: manjula.simiyon@yahoo.co.in

How to cite this article: Joju S, Simiyon M, Thilakan P, et al. A Qualitative Analysis of Patients' Experiences during their Pathway to Psychiatric Care. Ind J Priv Psychiatry 2019;13(2):37-43.

Source of support: PIMS student fellowship

Conflict of interest: None

In India, patients with mental illness are stigmatized invariably. Visiting the THs is less stigmatizing than visiting MHPs. The reasons given by patients for visiting THs according to Naik et al. ${ }^{9}$ were (1) prior encouraging results with faith-healing in self and others; (2) causality was alleged to be supernatural, which needed a religious healer rather than a doctor; and (3) as the symptoms are not seen as physical problems, a medical doctor was not considered. Also, these THs have high acceptability and accessibility as they are part of their local communities. ${ }^{2}$

Even though quantitative methods are the commonly used research methods, they may not be able to answer a few rare or unexplored phenomena related to human behavior. Qualitative studies can enhance our understanding of human behavior and health services. They provide a basic understanding of complex situations, which cannot be explained by quantitative methods. ${ }^{10}$ The use of qualitative methods is central to make progressive research in healthcare. ${ }^{11}$ This study was designed to understand the patients' experiences during the pathway to psychiatric care

() The Author(s). 2019 Open Access This article is distributed under the terms of the Creative Commons Attribution 4.0 International License (https://creativecommons. org/licenses/by-nc/4.0/), which permits unrestricted use, distribution, and non-commercial reproduction in any medium, provided you give appropriate credit to the original author(s) and the source, provide a link to the Creative Commons license, and indicate if changes were made. The Creative Commons Public Domain Dedication waiver (http://creativecommons.org/publicdomain/zero/1.0/) applies to the data made available in this article, unless otherwise stated. 
through qualitative research using a thematic analysis. Thematic analysis is a technique for classifying, analyzing, and recording patterns or themes within data. ${ }^{12}$

\section{Materials and Methods}

This hospital-based study was conducted to collect retrospective information from patients and their caretakers on their care-seeking pathways that lead them to the Department of Psychiatry of PIMS, Puducherry. After obtaining Institutional Ethical Clearance and written informed consent, 150 adult patients accompanied by primary caregivers attending the Department of Psychiatry for the first time in the 2 months (July and August 2018) period were selected through purposive sampling. Adult patients accompanied by caregivers who cannot reasonably participate in the research were excluded. Those who fulfill the inclusion criteria were interviewed using the proforma and the WHO encounter form. ${ }^{13}$

Patients who had more than two encounters were invited to participate in the qualitative interview. The current qualitative study aimed to elicit and gather perspectives in a subset of patients with mental illness and or their primary caretakers and understand their subjective experiences during their pathway to psychiatric care. The participants were encouraged to elaborate on the encounters they had, the path taken by them, and the experiences they had during their journey from the onset of illness until they reach our Department of Psychiatry. In those patients who were severely symptomatic, interviews were conducted only with the primary caretakers. Totally, 18 participants accepted to participate within the timeframe. A semi-structured guided interview format was used to gather their narratives. Interviews, which lasted between 45 minutes and 60 minutes, were transcribed and translated from audio recordings. The interviews were originally conducted in Tamil and took place in a separate room in the Psychiatric Outpatient Department of PIMS, ensuring safety, confidentiality, and privacy. To ensure participant validation, at the end of the interview, the summary of the interviews was read back to the participants.

\section{Analysis of Qualitative Data}

The narratives were read and re-read, and intensive coding was done to segregate themes. The data were retrieved, coded, and systematically organized according to patterns and themes. To minimize bias, independent coding was done. As no prior theoretical framework was chosen to explain, the inductive method was used to arrive at the themes. Codes were grouped into themes, which were then described, and any differences resolved by discussing within the research team. Pertinent subthemes were grouped under the themes. Redundant and repetitive themes were removed or regrouped.

\section{Results}

Table 1 depicts the details of the participants.

Even though 18 interviews were conducted, data reached theoretical saturation after analyzing 15 interviews.

Four major themes were extracted, which were further analyzed for subthemes.

- Clinical presentation and their understanding

- Encounters

- Expenditure during their pathway

- Remarks made by the patient/caregiver

\section{Clinical Presentation and their Understanding}

Under this theme, two themes were mined. Each theme is elaborated with an example

- The first symptom that triggered help-seeking behavior

- Attribution and understanding of the illness by the family

First Symptom that Triggered Help-seeking Behavior

Most of them started seeking help only when there was a severe symptom. The following were the most common reasons regarding the symptomatology after which the patient or the family sought help.

- Violence

- Irritability

- Suicidal attempt

- Complications related to substance use

For example, Case 30 "We had to take him as he became very violent and hit everyone at home."

Case 86 "He was irritable all the time and broke my new phone"

Case 33 "I attempted suicide thrice within a week...first with sleeping pills, then with Ujala, and then naphthalene balls...then I realized that I have some problem"

Case 92: "Few days after he stopped drinking, he became so restless and fearful. He could not recognize us. So his aunt told that he is possessed and advised us to take him to a godman"

Attribution and Understanding of the Illness by the Family

Patients and their family members had their explanatory models for the illness, which were colored by cultural beliefs. The same participants had different explanations as the disease progressed.

- Breakup of a relationship

- Watching excessive pornography/masturbation (single patient)

- Possession by evil spirit/occult practices

- Mental illness

For example, Case 126 "She was in love with a man. Later he refused to marry her. So, she became ill"

Case 24 "I also masturbated a lot. I was dull and tired. All my problems started then."

"But later I realized that I have problems in my mind".

Case 148 "We went to a temple thinking that it's because of evil spirit."

Case 92 "Few days after he stopped drinking, he became so restless and fearful. He could not recognize us. So his aunt told that he is possessed and advised us to take him to a godman"

\section{Encounters}

All the 18 patients had visited a TH at least once. Most of them had an average of 2 encounters.

\section{Encounters were Suggested by}

Most of the time caretakers followed the path suggested by others. These include

- Relatives

- Friends

- Neighbors

For example, Case 126 "My friends brought the godman to home"

Case 9 "Our neighbors took me to Chennai saying that if I do a remedy there, I will be all right." 
Table 1: Description of the participants of qualitative interview

\begin{tabular}{|c|c|c|c|c|c|c|}
\hline S. no. & Case no. & Diagnosis of the patient & Age/sex & $\begin{array}{l}\text { Relationship to the } \\
\text { participant }\end{array}$ & No. of encounters & $\begin{array}{l}\text { Duration of untreated } \\
\text { illness (in months) }\end{array}$ \\
\hline 1 & 9 & $\begin{array}{l}\text { Emotionally unstable personality } \\
\text { disorder with deliberate self-harm }\end{array}$ & $21 / F$ & Self & 8 & 12 \\
\hline 2 & 24 & Somatization disorder & $24 / \mathrm{M}$ & Self & 13 & 36 \\
\hline 3 & 30 & Alcohol dependence & $36 / \mathrm{M}$ & Wife & 3 & 84 \\
\hline 4 & 33 & Major depression & $22 / \mathrm{M}$ & Self & 11 & 0.5 \\
\hline 5 & 69 & Schizophrenia & $35 / M$ & Sister-in-law & 8 & 36 \\
\hline 6 & 78 & Schizophrenia with OCD & $32 / \mathrm{M}$ & Wife & 7 & 12 \\
\hline 7 & 86 & Schizophrenia & $59 / \mathrm{M}$ & Wife & 3 & 69 \\
\hline 8 & 92 & Alcohol dependence & $32 / \mathrm{M}$ & Wife & 2 & 240 \\
\hline 9 & 125 & Schizophrenia & $40 / \mathrm{M}$ & Mother & 3 & 72 \\
\hline 10 & 126 & Bipolar disorder & $28 / \mathrm{F}$ & Aunt & 6 & 40 \\
\hline 11 & 138 & Schizophrenia & $58 / F$ & Husband & 2 & 4 \\
\hline 12 & 140 & Schizophrenia & $18 / \mathrm{F}$ & Mother & 4 & 0.33 \\
\hline 13 & 148 & Major depression & $35 / F$ & Self & 2 & 6 \\
\hline 14 & 149 & Schizophrenia & $40 / \mathrm{F}$ & Mother & 4 & 84 \\
\hline 15 & 150 & Schizophrenia & $35 / \mathrm{F}$ & Mother & 4 & 24 \\
\hline
\end{tabular}

Case 78 "Then our relatives told He is fine only. Not ill. Don't spoil his health by giving tablets. Just take him to a temple."

\section{Encounters}

As already discussed, most of them had multiple encounters. Predominantly priests or godman seems to be popular. There were also instances when they met a medical practitioner, usually a general practitioner.

- Allopathic/alternative therapist

- GP

- Psychiatrist

- Ayurveda practitioner

- Siddha practitioner

For example, Case 9 "first I went to a nearby clinic only" Case 33 "On the way back from the temple, we saw a psychiatrist's house and went there"

Case 78 "As I was unable to eat I went to an Ayurvedic practitioner" Case 78 "We went to see the local doctor one year ago. He told that this is a mental illness and gave tablets"

- THs

The majority of the participants have visited TH multiple times. The following themes emerged when the encounters were analyzed in this aspect.

- Priest

- Godman

- Astrologer

- Soothsayers

- Hidharath (Muslim Priest)

For example, Case 92 "We went to a temple in Kalavan to see the priest"

Case 24 "Then I went to see a God-man"

Case 92 "One week ago, we went to the mosque and met the Imam" Case 78 "Then we went to an astrologer"

\section{Explanations and Remedies Suggested by the Healers}

Patients could recall the explanations given by both the medical and non-medical healers. Sometimes, they also felt that enough explanations were not given. One of the practitioners has told a female pregnant woman that these mood swings are physiological.

- Explanations were given by the allopathic/alternative therapist

- Labeled it as mental illness

- Normal physiological mood swings.

For example, Case 78 "We went to a local doctor one year ago. He told that this is a mental illness and gave tablets. He didn't explain further. But after taking tablets he was better"

Case 126 "Then the doctor told that she (patient) is having these mood swings because of her pregnancy and she will be all right after delivery"

- Remedies prescribed by the allopathic/alternative therapist: When these patients visited the doctors, they suggested various remedies. Most of the time they have not followed the suggestions.

- Pills

- Counseling/psychotherapy

- In-patient care

- Electroconvulsive therapy

- Brain scan

For example, Case 33 "They spoke to me and gave me some medications and advised me to come for counseling weekly"

Case 126 "They gave tablets and shock treatment"

Case 86 "They said that he needs to be admitted"

- Reasons for poor compliance

Even though they had visited a hospital or a psychiatric setup, most of them were poorly compliant. The reasons related to poor compliance as follows:

- No immediate improvement

- Cost

- Lack of insight

- Relatives discouraging medical treatment

- Side effects/pregnancy and fear of teratogenicity

For example, Case 125 "But he did not take the medication properly... and when he took he was better. But when he stopped he was worse" 
Case 86 "They said that he needs to be admitted and it will cost around Rs. 20,000. But I can't pay that much hence opted for OP treatment with tablets. That itself came around Rs. 700 per month. My daily wage is only Rs. 120. So I could not follow-up there."

Case 138 "She took the tablets for 6 months and had a lot of side effects. So, stopped it"

Case 33 "As I was feeling better I didn't take medicine or attend counseling. But again, I started crying excessively."

Case 78 "Then our neighbors told he is fine only. Not ill. Don't spoil his health by giving tablets. Just take him to a temple."

Case 138 "One of the church members told her that let her faith not be upon the tablets but only upon God; hence she stopped the tablets"

Case 150 "Then I became pregnant. I wanted my child to be normal so / stopped medications."

- Explanations are given by the THs

The most interesting part of the study is the exploration of the TH's attributions. These include the following. Most of them have given a magico-religious explanation except one who suggested the possibility of mental illness.

- Possessed by evil spirit/ghost/God

- Result of black magic/sorcery

- Practice of burning the menstrual clothes

- Scared after seeing something

- Bad timing/horoscope/bad omen

- Disease (one TH)

For example, Case 30 "He told that he is possessed"

Case 9 "They told that someone has done black-magic against me"

Case 9 "Another god-man told that my menstruation clothes were burnt by my family members and that's why l am having this problem"

Case 86 "A 50-year-old woman, a sooth-sayer told me that my husband has God upon him, so someone would have done some sorcery against him or this could be a disease also"

Case 92 "He said that she got scared of something and possessed by a ghost"

Case 78 "They'd asked us to go to an astrologer. And he told that it's due to bad timing of Raahu and Kethu."

- Remedies prescribed by the THs

Though most of the priests or godman belonged to one particular religion, the remedies prescribed by them varied widely.

- Animal/bird sacrifice

- Rituals in the graveyard at midnight

- Poojas using various ingredients

- Growing yagna

- Physical assault

- Tying amulet

- Changing houses

- Drinking holy water or eating holy ash

For example, Case 30 "He asked my husband to promise that he won't drink again and threatened him that if he drinks again he will have blood vomiting and die. He also sacrificed a hen and did pooja with its blood"

"He told that he is possessed and hit him badly. They asked him to leave the house."

Case 9 "They packed the holy ash in a leaf and asked me to eat it." "He plucked my hair and put multiple knots in it. It started bleeding"

Case 125 "That god-man killed a hen and made me drink its blood. Later he dug a hole near the temple entrance, buried the hen there and covered it with a lemon"
Case 86 "But the godman said that I have to change my house as omen was not good with the current house"

"They tied an amulet and sent us."

Case 25 "They asked me to buy hen, fish, and arrack. Then they took me to a graveyard and performed some rituals."

Case 33 "There they said that they have to grow a "yagna" and we had to pay for the amulet. By doing the yagna they promised us that they could capture the ghost in a bottle"

"They beat me, slapped on my face, plucked my hair, and cut my plait, cut all the amulets I had, they forcefully fed me with banana and holy ash."

"They kept calling us again saying that some ritual has to be done at the entrance of our house or else that dead girl would come and possess me."

Case 92 "She said that we should buy 5 types of millets. She chanted over it...carried it around the head few times and threw it off"

Case 78 "They asked us to buy winnow sieve, garlic, pumpkin, egg, lemon, clothes, dhoti, arrack, camphor, rice, sambar, etc. We bought around 35 things"

Case 92 "Then we went to an Imam, He chanted and gave a bottle of water"

Case 69 "He also asked us to buy a doll, dress it up, take a pumpkin around the doll and then break it"

\section{Expenditure During the Pathway}

\section{Expenditure}

Another important finding was the cost spent by patients for these remedial measures.

- It ranged between INR-3000 and INR-6,00,000. Among these only 3 patients had spent on allopathic/alternative care. The rest was spent on traditional healing practices.

- The other related theme was the source of money spent on these remedies.

- Borrowing money

- Pledging jewelry

- using scholarship money (one patient)

For example, Case 86 "I borrowed money from many people and took him to astrologers. I have pledged all my jewels in a pawn shop and spent for him."

Case 24 "I spent all the scholarship money I got."

\section{Remarks Made by the Patients Regarding the Healers/Treatment}

During the interview, the participants expressed their opinion about the healers they had met...

\section{Regarding Medical/Alternative Therapist and their Suggestions}

- Expensive

- Unfriendly

- Did not give enough explanations

For example, Case 86 "Then we took him to hospital. They said that he needs to be admitted and asked me to pay Rs. 20,000 immediately as advance"

Every month it cost a few thousand for tablets alone. I couldn't afford.

Case 33 "He scolded me very badly saying why are you thinking about that man and troubling your parents?" 
Case 76 "He told me that this is a mental illness and gave tablets. He didn't explain further."

\section{Regarding THs and their Suggestions}

Patients had neutral to a negative attitude toward the rituals and remedies.

For example, Case 9"They told me that someone has done blackmagic against me! I didn't believe and told that there is no chance!"

Case 138 "We should believe in God but at the same time, we should take medicines as well. But he told me not to take tablets which were wrong."

Case 78 "But later we realized that all the explanations they gave us were nothing but lies."

Case 33 "My father didn't want to take me there, as they were beating me mercilessly"

Case 92 After drinking that holy water, there was some improvement that he started sleeping. But only for a few days.

\section{Summary of Qualitative Results}

Flowchart 1 explains the themes related to their pathway and Flowchart 2 about their encounters.

\section{Discussion}

Most of the cultural practices are reiterated from the themes and subthemes found in the study. Patients with mental illness are taken for help only when they have severe symptoms such as harm to self or others. Their understanding of mental illnesses and attributions typically reflected cultural beliefs. Only one person acknowledged that this could be a mental illness.

Treatment initiatives were taken by others most of the time. The encounters with magicoreligious treatment posed a unique challenge due to overlapping terms such as Pujari, Samiyar, and
Saadhu. Each has differences in their practices. According to Trivedi and Jilani, ${ }^{3}$ common factors that are associated with seeking help from THs are:

- Previous successful exposure to faith healing,

- Faith in supernatural healing may run in families,

- Monetary constraints,

- The apprehension of social stigma in being labeled,

- Easy accessibility of faith healers,

- Failure of medical care,

- Influence of neighbors and friends, and

- Lower socioeconomic status.

Though most of these factors apply to our patients as well, the financial aspects need special consideration. Though we could not make an appropriate estimate of the cost, patients had spent more on natural healing pathways than with allopathic/alternative care. Borrowing and pledging jewels, etc., which are unhealthy ways of handling finances, managed the financial needs.

The explanations and remedies given by the native healers are similar to the one found by Schoonover et al. ${ }^{7}$ and Kurihara et al. ${ }^{14}$ They attributed it to the evil forces and tried to nullify it by doing various rituals. Sometimes, violent and horrific methods were used. They have hit, verbally abused, pulled hair and made them drink blood. Also, the list of objects they had to purchase to do the rituals varied from five to thirty-five. Most (10/15) of them had asked for alcohol. Some remedies were very expensive such as changing the house. Doing rituals in the graveyard and growing yagna were also common practices as a remedy. Many patients have visited multiple faith healers and obliged to their instructions piously. A similar scenario was found in Bali as well. ${ }^{14}$ However, the same patients did not take the medications as advised. This may be related to the factor of fear or reverence associated with the THs. Schoonover et al. ${ }^{7}$ found that people consider them next to God. But the respect they had for doctors is visibly decreasing.

Flowchart 1: Major themes extracted from the qualitative analysis

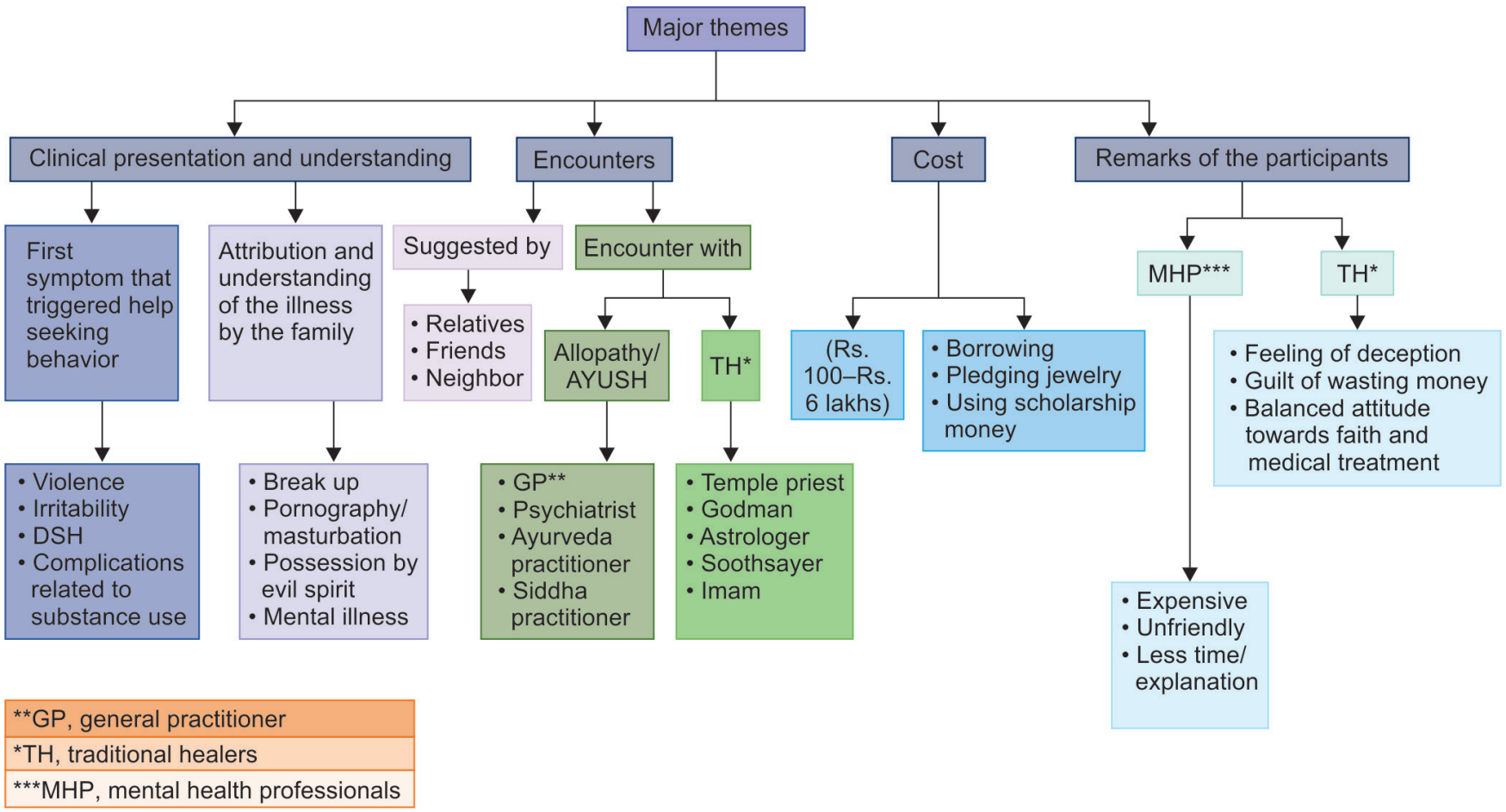


Flowchart 2: Themes related to the encounters

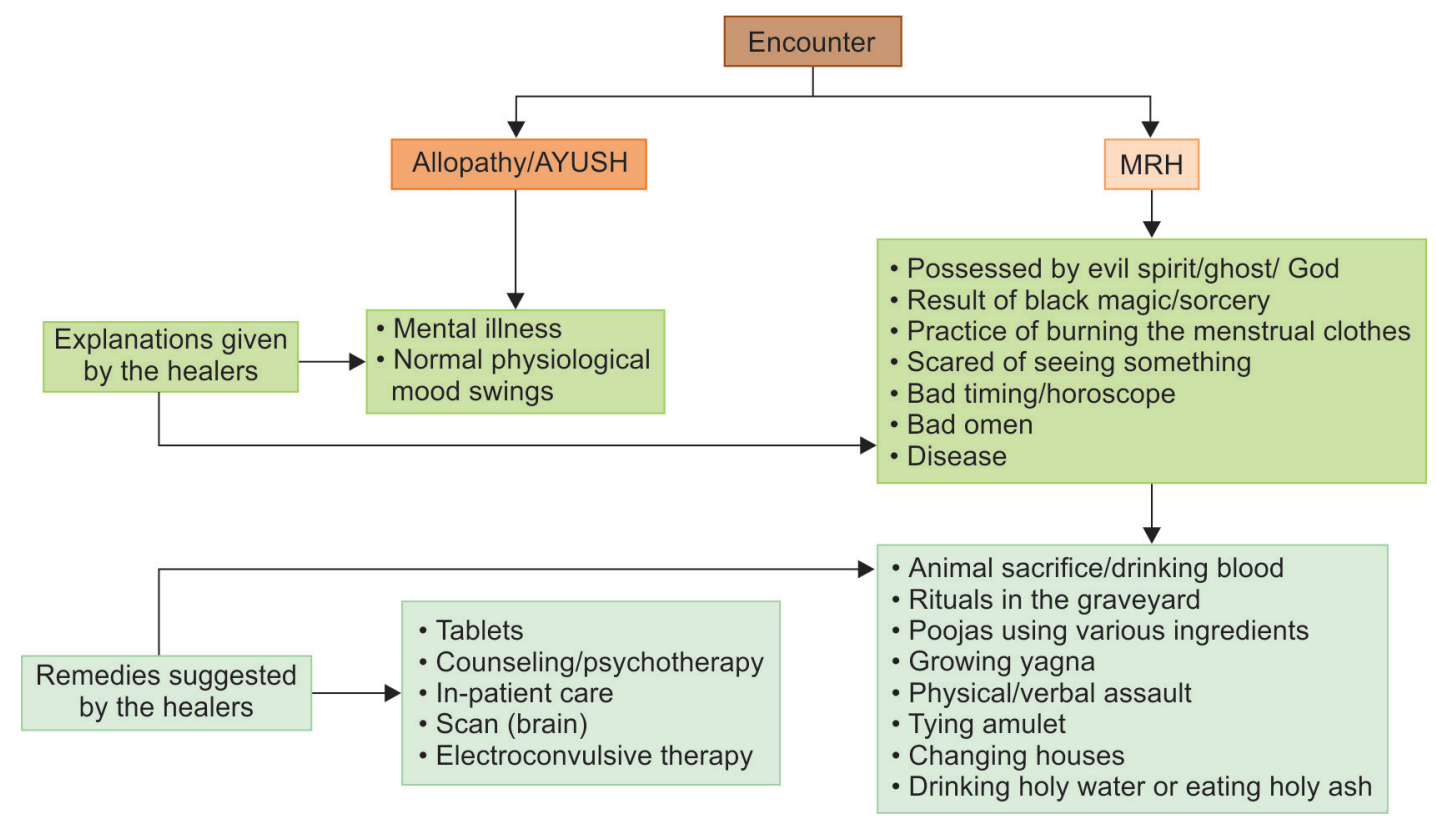

The encounter with a psychiatrist or medical practitioner was less frequent in this subgroup. Surprisingly, one of the medical practitioners had tried to normalize the mood symptoms probably causing a delay in seeking further care. This awakens us to the reality of other practitioner's knowledge about mental illness. Whenever they have met a psychiatrist, the standard of care was followed.

Even though we had not asked, one of the subthemes that was identified, the final remark they made about the TH. Most of the patients had developed a negative attitude toward the magico-religious healing practices. This may be due to the bitter experiences they had or failure to relieve symptoms. Few of them regretted spending a huge amount of money.

World Health Organization has already mentioned that these alternate pathways may hinder access to mental health services because of low awareness of available services, insufficient connections between services, a dearth of knowledge among rural populations about the causes of and treatments for mental disorders resulting in the underutilization of mental health services, insufficient mental health training of general practitioners and THs adding to low rates of detection, treatment, and referral of mental disorders in traditional and primary care settings, and so on. These hurdles frequently lead to undesirable outcomes, such as delays in pursuing care until full-blown disorders have established with a higher probability of unfavorable long-term outcomes and huger expenses of treatment.

In a country like India where cultural relativism is very important, we do agree that we have to respect the cultural explanatory model given by the patients, ${ }^{8}$ but $\mathrm{WHO}^{2}$ also has recognized that some of the traditional healing practices were associated with the human rights violation, as seen in our study participants. In our study also, we found that patients with serious conditions such as delirium tremens were considered to be possessed and taken to temple, which could have been life-threatening. Hence, cultural relativism cannot be preserved at the cost of the safety of the patient.

Bathla et al. ${ }^{15}$ has suggested that attempts should be made in every region to drive these godmen away from our society and to create knowledge about mental illnesses. In our view, we cannot completely remove the traditional healing system from the pathway, as it has become part of the collective subconscious of our culture ${ }^{15}$ and ingrained in our society for centuries. The community may not accept such measures. Rather, gaining their trust and working closely with THs would give us a better opportunity to gain acceptance from the community and modify certain harmful practices they have been performing. ${ }^{16}$

The Dawa-Dua model of Erwadi village of Tamil Nadu has paved a way forward for such efforts. Even though this village became popular after the tragic fire accidents of mentally ill persons in 2001, the religious leaders in Erwadi opened up to the idea of combining medical treatment with traditional healing. And in 2014, Erwadi Dargah incorporated a clinic run by the district mental health program within its campus and started offering its visitors access to professional psychiatric care, diagnosis, treatment, and medication. Patients were encouraged to continue their prayers alongside professional medical treatment and return for follow-up visits to improve their well-being. ${ }^{17}$ Other religious places popularly known for mental health remedies should also be incorporated in such a plan.

\section{References}

1. Gururaj G, Varghese M, Benegal V, et al. National mental health survey of India, 2015-16: Summary. Bengaluru: National Institute of Mental Health and Neurosciences; 2016.

2. Funk M, Saraceno B, Pathare $S$, , World Health Organization, editors. Organization of services for mental health. Geneva: World Health Organization; 2003. p. 74. (Mental health policy and service guidance package).

3. Trivedi J, Jilani AQ. Pathway of psychiatric care. Indian J Psychiatry 2011;53(2):97-98. DOI: 10.4103/0019-5545.82530.

4. Jain N, Gautam S, Jain S, et al. Pathway to psychiatric care in a tertiary mental health facility in Jaipur, India. Asian J Psychiatr 2012;5(4): 303-308. DOI: 10.1016/j.ajp.2012.04.003.

5. Lahariya C, Singhal S, Gupta S, et al. Pathway of care among psychiatric patients attending a mental health institution in central India. Indian J Psychiatry 2010;52(4):333-338. DOI: 10.4103/00195545.74308 . 
6. Biswal R, Subudhi C, Acharya SK. Healers and healing practices of mental illness in India: the role of proposed eclectic healing model. J Health Res Rev 2017;4(3):89-95. DOI: 10.4103/jhrr.jhrr_64_17.

7. Schoonover J, Lipkin S, Javid M, et al. Perceptions of traditional healing for mental illness in rural Gujarat. Ann Glob Health 2014;80(2):96-102. DOI: 10.1016/j.aogh.2014.04.013.

8. Chowdhury A. Pathway to psychiatric care and cultural myth. Indian J Psychiatry 2012;54(4):395-397. DOI: 10.4103/0019-5545.104849.

9. Naik SK, Pattanayak S, Gupta CS, et al. Help-seeking behaviors among caregivers of schizophrenia and other psychotic patients: a hospitalbased study in two geographically and culturally distinct Indian cities. Indian J Psychol Med 2012;34(4):338-345. DOI: 10.4103/0253-7176. 108214.

10. Reid AJ. What we want: qualitative research. Promising frontier for family medicine. Can Fam Physician 1996;42:387-389, 397-400.

11. Secker J, Wimbush E, Watson J, et al. Qualitative methods in health promotion research: some criteria for quality. Health Educ J 1995;54(1):74-87. DOI: 10.1177/001789699505400108.
12. Braun V, Clarke V. Using thematic analysis in psychology. Qual Res Psychol 2006;3(2):77-101. DOI: 10.1191/1478088706qp063oa.

13. WHO encounter form.pdf.

14. Kurihara $T$, Kato $M$, Reverger $R$, et al. Pathway to psychiatric care in Bali. Psychiatry Clin Neurosci 2006;60(2):204-210. DOI: 10.1111/j.14401819.2006.01487.x

15. Bathla M, Chandna S, Bathla J, et al. Faith healers in modern psychiatric practice: results of a 4 years study. Delhi Psychiatry Journal 2015;18(1):6.

16. Alem $A$, Jacobsson $L$, Araya $M$, et al. How are mental disorders seen and where is help sought in a rural Ethiopian community? A key informant study in Butajira, Ethiopia. Acta Psychiatr Scand Suppl 1999;397:40-47. DOI: 10.1111/j.1600-0447.1999.tb10693.x.

17. Dawa-Dua: How medical treatment complements prayer for people with mental illness in India [Internet]. [cited 2019 Oct 14]. Available from: http://blogs.worldbank.org/endpovertyinsouthasia/dawa-duahow-medical-treatment-complements-prayer-people-mental-illnessindia. 